J. Austral. Math. Soc. 22 (Series A) (1976), 380-382.

\title{
CHARACTERIZATIONS OF A GENERALIZED NOTION OF COMPACTNESS
}

\author{
GEORGE H. BUTCHER and JAMES E. JOSEPH
}

(Received 20 October 1975; revised 12 November 1975)

\begin{abstract}
This paper gives theorems which encompass known characterizations of many of the generalized compactness properties.
\end{abstract}

\section{Introduction}

Several generalized compactness properties of topological spaces have been characterized by analogues of the following theorems for compactness:

*) A space $X$ is compact if and only if the projection $\pi_{y}: X \times Y \rightarrow Y$ is closed for every space $Y$, Mrówka (1959), Hanai (1961), Franklin and Sorgenfrey (1966), Scarborough (1969).

**) A $T_{1}$ space $Y$ is compact if and only if each function into $Y$ with a closed graph (closed-graph function) is continuous, Franklin and Sorgenfrey (1966), Scarborough (1969), Kasahara (1973).

It has also been shown that $m$-compact spaces have characterizations with these forms, Hanai (1961), Franklin and Sorgenfrey (1966).

More recently, the generalized compactness properties, Lindelö, $m$ Lindelöf, $H$-closed and nearly-compact have been shown to have characterizations with these forms, Joseph (submitted for publication), Herrington and Long (1975), Joseph (to appear).

Also of recent interest has been the class of $(m, n)$-compact spaces (Hodel and Vaughan (1974), Vaughan (1975)). In this paper, we establish that $(m, n)$-compact spaces have characterizations with forms $(*)$ and $(* *)$. The class of $(m, n)$-spaces dates back to the work of Aleksandrov and Urysohn (1929) and has been extensively studied by Smirnov (1950) and independently by Gál $(1957,1958)$.

\section{Preliminary definitions and remarks}

A space is defined to be $(m, n)$-compact for the cardinals $m$ and $n$ if each open covering $\theta$ of the space with $|\theta|$ (the cardinality of $\theta$ ) at most $n$ contains 
a subcovering of the space with cardinality at most $m$. Using the convention that 1 stands for "finitely many", $\infty$ stands for "arbitrary cardinality" and $\omega$ stands for the first infinite cardinal, we see readily that the $(1, \infty)$-compact, $(1, m)$-compact, $(\omega, \infty)$-compact, and $(m, \infty)$-compact spaces are respectively the compact, $m$-compact, Lindelöf and $m$-Lindelöf spaces. Gál (1958) called a filter on a set $X$ an $(m, n)$-filter if the filter has a base with cardinality at most $n$ and with the $m$-intersection property; he proved that a space $X$ is $(m, n)$-compact if and only if every $(m, n)$-filter adheres to some point in $X$. We make the following definition.

Definition. A topological space is an $(m, n)$-space if 1) at each point of the space, there is an open set base of cardinality at most $n$, and 2) the intersection of any collection of at most $m$ open subsets of the space is open.

We will denote the closure of a subset $K$ of a topological space by $\mathrm{cl}(K)$.

\section{Main results}

We now give our main results.

Theorem 1. A topological space $X$ is an $(m, n)$-compact space if and only if $\pi_{y}: X \times Y \rightarrow Y$ is a closed function for every $(m, n)$-space $Y$.

Proof. Let $K \subset X \times Y$ be closed; let $y \in \operatorname{cl}\left[\pi_{y}(K)\right]$. Let $\theta$ be an open set base at $y$ with $|\theta| \leq n$. Then $\left\{c l\left[\pi_{x}(K \cap(X \times V))\right]: V \in \theta\right\}$ is a base for an $(m, n)$-filter on $X$. Let $x \in X$ satisfy $x \in \operatorname{cl}\left[\pi_{x}(K \cap(X \times V))\right]$ for each $V \in \theta$. It is not difficult to show that $(x, y) \in \operatorname{cl}(K)=K$ and consequently that $y \in \pi_{y}(K)$ to complete one part of the proof. For the converse, let $\mathscr{B}$ be a base for an $(m, n)$-filter on $X$ and let $y_{0} \notin X$. Let $Y=X \cup\left\{y_{0}\right\}$ with the topology generated by $\{\{y\}: y \in X\} \cup\left\{B \cup\left\{y_{0}\right\}: B \in \mathscr{B}\right\}$. Then $Y$ is an $(m, n)$ space. Let $K=\{(y, y): y \in X\}$. Then $y_{0} \in \operatorname{cl}\left(\pi_{y}(K)\right)=\pi_{y}[\mathrm{cl}(k)]$. Let $x \in X$ with $\left(x, y_{0}\right) \in \operatorname{cl}(K)$ and let $V$ be open in $X$ about $x$. Then $[V \times(Y-V)] \cap$ $K=\varnothing$, so $Y-V$ is not open in $Y$. Thus $B \cap V \neq \varnothing$ is satisfied for each $B \in \mathscr{B}$ and $\mathscr{B}$ adheres to $x$. This completes the proof.

Theorem 2. A $T_{1}$ space $Y$ is $(m, n)$-compact if and only if all closedgraph functions (bijections) from $(m, n)$-spaces to $Y$ are continuous.

Proof. Let $X$ be an $(m, n)$-space, suppose $Y$ is $(m, n)$-compact and assume $g: X \rightarrow Y$ is a closed-graph function. If $G(g)$ is the graph of $g$ and $K \subset Y$ is closed, then $\pi_{y}^{-1}(K) \cap G(g)$ is closed in $X \times Y$ and $g^{-1}(K)=$ $\pi_{x}\left[\pi_{y}^{-1}(K) \cap G(g)\right]$ which is closed in $X$ by Theorem 1 ; so one part of the theorem is proved. For the converse, suppose all closed-graph functions from $(m, n)$-spaces to the $T_{1}$ space $Y$ are continuous, let $x_{0} \in Y$, and let $\mathscr{B}$ be a 
base for an $(m, n)$-filter on $Y$ which fails to adhere to any point in $Y-\left\{x_{0}\right\}$. Let $X=Y$ with the topology generated by $\left\{\{x\}: x \in X=\left\{x_{0}\right\}\right\} \cup$ $\left\{B \cup\left\{x_{0}\right\}: B \in \mathscr{B}\right\}$ as base. Let $i: X \rightarrow Y$ be the identity function and let $(x, y) \in(X \times Y)-G(i)$. If $x \neq x_{0}$, then $\{x\}$ is open in $X, Y-\{x\}$ is open about $y$ in $Y$ and $(\{x\} \times(Y-\{x\})) \cap G(i)=\varnothing$, so $(x, y) \notin \operatorname{cl}(G(i))$. If $x=x_{0}$, then $y \neq x_{0}$, so there is a $V$ open in $Y$ about $y$ and a $B \in \mathscr{B}$ satisfying $B \cup\left\{x_{0}\right\} \subset X-V$. So $X-V$ is open in $X$ about $x_{0}$ and $((X-V) \times V) \cap$ $G(i)=\varnothing$; thus $(x, y) \notin \mathrm{cl}(G(i)), i$ is a closed-graph bijection and $i$ is continuous since $X$ is an $(m, n)$-space. Let $V$ be open in $Y$ about $x_{0}$. Then $i^{-1}(V)$ is open in $X$ about $x_{0}$, so there is a $B \in \mathscr{B}$ satisfying $B \subset V$ and $\mathscr{B} \rightarrow x_{0}$. This completes the proof.

\section{References}

P. Aleksandrov and P. Urysohn (1929), 'Mémoire sur les espaces topologiques compacts', Nederi. Akad. Wetensch Proc. Ser. A 14, 1-96.

I. S. Gál (1957), 'On a generalized notion of compactness, I', Nederl. Akad. Wetensch Proc. Ser. A 60, 421-430.

I. S. Gál (1958), 'On the theory of ( $m, n)$-compact topological spaces', Pacific $J$. Math. 8, 721-734.

S. P. Franklin and R. H. Sorgenfrey (1966), 'Closed and image-closed relations', Pacific J. Math. 19, 433-439.

S. Hanai (1961), 'Inverse images of closed mappings, I', Proc. Japan Acad. 37, 298-301.

L. L. Herrington and P. E. Long (1975), 'Characterizations of H-closed spaces', Proc. Amer. Math. Soc. 48, 469-475.

R. E. Hodel and J. E. Vaughan (1974), 'A note on $[a, b]$-compactness', Gen. Top. and Appl. 4, 179-189.

J. Joseph (to appear), 'On $\boldsymbol{H}$-closed spaces', Proc. Amer. Math Soc.

J. Joseph (to appear), 'Characterizations of nearly-compact spaces', Boll. Un. Mat. Ital.

J. Joseph (submitted for publication), 'Characterizations of $\boldsymbol{m}$-Lindelöf spaces'.

S. Kasahara (1973), 'Characterizations of compactness and countable compactness', Proc. Japan Acad. 49, 523-524.

S. Mrowka (1959), 'Compactness and product spaces', Colloq. Math. 7, 19-22.

C. T. Scarborough (1969), 'Closed graphs and closed projections', Proc. Amer. Math. Soc. 20, $465-470$.

Yu. M. Smirnov (1950), 'On topological spaces, compact in a given interval of powers', Akad. Nauk. SSSR Izvest. Ser. Mat. 14, 155-178.

J. E. Vaughan (1975), 'Convergence, closed projections and compactness', Proc. Amer. Math. Soc. 51, 469-475.

J. E. Vaughan (1975), 'Some properties related to [a, b]-compactness', Fund. Math. 87, 251-260.

Department of Mathematics,

Howard University,

Washington, D. C.

Department of Mathematics,

Federal City College,

Washington, D. C. 